\title{
Prognostic significance of VEGF expression in patients with bulky cervical carcinoma undergoing neoadjuvant chemotherapy Chel Hun Choi ${ }^{\dagger 1}$, Sang Yong Song ${ }^{\dagger 2}$, Jung-Joo Choi ${ }^{1}$, Young Ae Park ${ }^{1}$, Heeseok Kang1, Tae-Joong Kim¹, Jeong-Won Lee ${ }^{1}$, Byoung-Gie Kim¹, Je- Ho Lee ${ }^{1}$ and Duk-Soo Bae*1
}

\author{
Address: ${ }^{1}$ Department of Obstetrics and Gynecology, Samsung Medical Center, Sungkyunkwan University School of Medicine, Seoul, Korea and \\ ${ }^{2}$ Department of Pathology, Samsung Medical Center, Sungkyunkwan University School of Medicine, Seoul, Korea \\ Email: Chel Hun Choi - Chelhun.choi@samsung.com; Sang Yong Song - yoda.song@samsung.com; Jung- \\ Joo Choi - jungjoo.choi@samsung.com; Young Ae Park - youngae1025.park@samsung.com; Heeseok Kang - hsdr.kang@samsung.com; Tae- \\ Joong Kim - tj28.kim@samsung.com; Jeong-Won Lee - garden.lee@samsung.com; Byoung-Gie Kim - bksong.kim@samsung.com; Je- \\ Ho Lee - jehojeho.lee@samsung.com; Duk-Soo Bae* - huna0@naver.com \\ * Corresponding author †Equal contributors
}

Published: II October 2008

BMC Cancer 2008, 8:295 doi:10.1 186/147/-2407-8-295

This article is available from: http://www.biomedcentral.com/I47I-2407/8/295

(c) 2008 Choi et al; licensee BioMed Central Ltd.

This is an Open Access article distributed under the terms of the Creative Commons Attribution License (http://creativecommons.org/licenses/by/2.0), which permits unrestricted use, distribution, and reproduction in any medium, provided the original work is properly cited.

\begin{abstract}
Background: The prediction of response to treatment would be valuable for managing cervical carcinoma with neoadjuvant chemotherapy.

Methods: To this end, the expression of VEGF was analyzed by immunohistochemistry using paraffin-embedded pre-treatment cervical biopsy tissues. This study included 29 patients with bulky IB to IIA cervical squamous cell carcinoma treated with neoadjuvant chemotherapy.
\end{abstract}

Results: Fifteen (5I.7\%) of 29 patients were scored as VEGF-positive. Response to chemotherapy (complete response or residual tumor with less than $3 \mathrm{~mm}$ stromal invasion) was observed in eight patients $(27.6 \%)$, and it was negatively associated with VEGF expression $(P=0.009)$. With logistic regression analysis, VEGF positivity continued to be an independent predictor for poor response $(P=0.032)$. In addition, the progression-free survival rate was significantly lower in patients with VEGF-positive tumors $(P=0.033)$.

Conclusion: Pretreatment assessment of VEGF expression may provide additional information for identification of patients with cervical cancer who had a low likelihood of response to neoadjuvant chemotherapy and an unfavorable prognosis.

\section{Background}

Carcinoma of the uterine cervix is the second most common cancer in women, but the prognosis remains very poor in bulky or locally advanced disease [1]. Although concurrent chemoradiation (CCRT) is now considered standard treatment, neoadjuvant chemotherapy (NAC) has been adopted to improve the prognosis for these cases
$[2,3]$. The development of convenient and reliable biomarkers predicting the treatment response would be valuable for patient management. If non-responsive tumors could be identified before NAC, using predictive biological factors, these patients could be allocated to CCRT. Furthermore, it would be reinforced if the biological factors found do not affect the response to CCRT. 
The correlation of angiogenesis with either metastasis or a poor prognosis has been reported in various cancers [4-6]. Among the angiogenic factors, vascular endothelial growth factor (VEGF) has been shown to have a pivotal role in tumor angiogenesis. However, the correlation between VEGF expression and prognosis in patient with cervical cancer has been inconsistent; this may be because of the marked heterogeneity of patient disease stages and treatment modalities in reported studies [7-9]. Although, there are some reports that show that VEGF plays an important role in patient response to chemotherapeutic agents, [10] there is little information available on its predictive value for treatment response in patients receiving NAC for cervical carcinoma. Therefore, we evaluated whether VEGF may have predictive value for patient response to NAC in cases with bulky cervical carcinoma.

The aim of the present study was to investigate the expression of VEGF and their possible role as predictors of response to NAC in patients with bulky cervical carcinoma.

\section{Methods}

\section{Patients and samples}

Of the patients with locally advanced cervical carcinoma, who had presented to the Samsung Medical Center, 46 patients with stage IB2 to IIB enrolled into a phase II trial of NAC [11]. Among them, 29 patients with stage IB2 to IIA and squamous cell histology were selected to minimize heterogeneity of the patient population studied (15 patients with stage IIB and 2 patients with adenocarcinoma were excluded). The Institutional Review Board at
Samsung Medical Center (Seoul, Korea) approved the protocol, and all patients provided written informed consent before entry into the trial. None of the patients was pretreated with any other chemotherapy or radiotherapy before the NAC. The median patient age was 47 years (range, 33 to 70 ). Twenty (69.0\%) patients had stage IB2 disease and nine $(31.0 \%)$ patients had stage IIA. The other clinicopathologic characteristics are shown in Table 1.

\section{Treatment and response}

Cisplatin-based chemotherapy (combination of vincristine $1 \mathrm{mg} / \mathrm{m}^{2}$, mitomycin-C $10 \mathrm{mg} / \mathrm{m}^{2}$ and cisplatin 75 $\mathrm{mg} / \mathrm{m}^{2}$ ) was administered every 3 weeks [11]. A type III radical hysterectomy with pelvic and paraaortic lymph node dissection was performed within 3 weeks of the administration of the third cycle of NAC in all patients. Following radical surgery, adjuvant radiotherapy was performed if lymph node metastasis, parametrial involvement or a positive resection margin were found.

In this study, the tumor response was evaluated pathologically. Complete response (CR) was defined as a complete disappearance of the invasive tumor in the cervix with negative nodes, and optimal pathologic response (OPR) was defined as a residual tumor with less than $3 \mathrm{~mm}$ stromal invasion. The 3-mm threshold used was chosen because it represents the maximal extension of FIGO stage IA1 cervical tumor, which is usually considered cured after local resection. And the role of OPR as a possible surrogate endpoint for survival in the neoadjuvant setting, has been reported [12]. In the present study, patients with CR or OPR were grouped together as responders.

Table I: Immunoreactivity of VEGF according to clinicopathologic characteristics of the cervical carcinoma patients

\begin{tabular}{|c|c|c|c|c|}
\hline \multirow[b]{2}{*}{ Variables } & \multirow[t]{2}{*}{ No. of patients } & \multicolumn{2}{|c|}{ VEGF expression } & \multirow[b]{2}{*}{$P$} \\
\hline & & Positive & Negative & \\
\hline Total & 29 & 15 & 14 & \\
\hline \multicolumn{5}{|l|}{ Age } \\
\hline$\geq 50$ years & 12 & 7 & 5 & 0.55 \\
\hline$<50$ years & 17 & 8 & 9 & \\
\hline \multicolumn{5}{|l|}{ Stage } \\
\hline IB2 & 20 & 9 & II & 0.28 \\
\hline IIA & 9 & 6 & 3 & \\
\hline \multicolumn{5}{|c|}{ Cervical tumor size } \\
\hline$\geq 5 \mathrm{~cm}$ & 14 & 9 & 5 & 0.19 \\
\hline$<5 \mathrm{~cm}$ & 15 & 6 & 9 & \\
\hline \multicolumn{5}{|c|}{ Clinical node involvement } \\
\hline Yes & 11 & 7 & 4 & 0.32 \\
\hline No & 18 & 8 & 10 & \\
\hline \multicolumn{5}{|l|}{ SCC Ag level } \\
\hline$\geq 5 \mathrm{ng} / \mathrm{ml}$ & 14 & 8 & 6 & 0.57 \\
\hline$<5 \mathrm{ng} / \mathrm{ml}$ & 15 & 7 & 8 & \\
\hline
\end{tabular}

$\mathrm{a}(\mathrm{n}=29)$

aThe $P$-value was determined using Chi-square test or Fisher's exact test. 


\section{Immunohistochemistry and evaluation}

Paraffin-embedded tissue blocks of formalin-fixed cervical biopsy specimens taken pre-treatment, were processed for conventional histological assessment by hematoxylin and eosin (H\&E) staining and immunohistochemical (IHC) analysis using the avidin - biotin - peroxidase method. VEGF protein expression was detected by mouse anti-human monoclonal VEGF (ab1316) antibody (Abcam, Inc., Cambridge, UK), using conventional peroxidase methods [13]. In brief, $4 \mu \mathrm{m}$ thick sections were deparaffinized in xylene, dehydrated through graded alcohol concentrations and incubated in citrate buffer $(\mathrm{pH}=$ 6.0) for 5 min using a household microwave oven at 800 $\mathrm{W}$. After microwave exposure, the slides were allowed to cool to room temperature. The slides were briefly washed with PBS and incubated for 15 min with 3\% hydrogen peroxide in methanol to block endogenous peroxidase activity. The antibody to VEGF was diluted 1:100 and incubated for $1 \mathrm{~h}$ at room temperature. Biotinylated antimouse/rabbit antibodies (DAKO) at a dilution of 1:500 were used as the second antibody. Negative controls included substitution of the monoclonal antibody with normal mouse IgG of the same concentration as the monoclonal antibody [see Additional file 1]. Sections of corpus luteum were used as positive control for VEGF immunostaining. After washing, ABC (DAKO) was applied and diaminobenzydine was used for visualization. Tissue sections were lightly counterstained with hematoxylin and then examined by light microscopy.

Assessment of the staining was scored independently by two investigators (SYS and CHC) without knowledge of the clinicopathological findings. Expression was defined as positive if distinct staining of the cytoplasm was observed in at least $10 \%$ of tumor cells [14]. The scoring by the two investigators was similar. In the cases of disagreement, slides were reevaluated and discussed until consensus was achieved.

\section{Statistical analysis}

Fisher's exact probability test or the Chi-square test was used to analyze frequency data. Multiple logistic regression models were used to identify independent prognostic factors for patient response. The tumor stage, nodal involvement, tumor size, and SCC-Ag levels were entered into the logistic regression models. Disease-free survival was measured by the Kaplan-Meier method. Differences between groups were tested using the log-rank test. To determine the independent prognostic value for patient disease-free survival, a Cox regression model was constructed using tumor stage, LN involvement, tumor size, SCC-Ag level, and IHC status as covariates. A P-value of less than 0.05 was considered significant. SPSS 10.0 (SPSS Inc., Chicago, IL) was used for the statistical analysis.

\section{Results}

Figure 1 show representative results of IHC staining. Fifteen $(51.7 \%)$ of 29 patients were scored as VEGF-positive (Table 1). Table 1 lists the positivity of the proteins according to clinicopathologic characteristics.

Pathologic responses, including complete and optimal responses, were observed in eight $(27.6 \%)$ patients, with no response in the remaining $21(72.4 \%)$ patients (Table $2)$. VEGF expression was shown to be highly associated with tumor susceptibility to NAC. The response rate of VEGF-positive tumors was significantly lower than VEGFnegative tumors $(7 \%$ vs. $50 \%, P=0.009)$. When logistic regression was applied, VEGF positivity continued to be an independent predictor of poor response to treatment $(P=0.032)$.

With a median follow-up period of 48 months (range, 3 to 105$)$, three $(10.3 \%)$ of 29 patients died of disease and recurrence occurred in seven $(24.1 \%)$. The overall 5-year disease-free survival rate was higher in the responder group than the non-responder group (100\% vs. 65\%), although this difference was not statistically significant $(P$ $=0.07$ ) (Figure 2A). The progression-free survival rate was significantly lower in patients with VEGF-positive tumors (vs. VEGF-negative tumors, $P=0.033$ ) (Table 3 and Figure 2B). VEGF-positivity was identified as an independent predictor of patient disease-free survival using a Cox regression model ( $P=0.037$; hazards ratio, $11.4 ; 95 \% \mathrm{CI}$, 1.15 - 112.58) (Table 3).

\section{Discussion}

In this study, the clinical significance of IHC-positivity for VEGF in pre-treatment biopsy specimens was examined in 29 patients with cervical squamous cell carcinoma undergoing NAC. The most interesting finding of our study was the strong correlation between VEGF expression and response to NAC. These results suggest that patients with cervical cancer who are positive for VEGF expression are less likely to benefit from NAC. Therefore, patient monitoring for VEGF expression may provide an important determinant for the differential treatment of bulky cervical cancer. Although it is very difficult to develop the best alternative strategy in such VEGF-positive patients, concurrent chemoradiation or radical surgery without delay or the addition of anti-VEGF therapy may be useful in improving the prognosis of those patients [15]. However, additional study is needed for confirmation of these findings.

The reason for the correlation between VEGF positivity and chemoresistance is unclear. The presence of increased vascularity may suggest improved tumor oxygenation and drug delivery; this may improve response to chemotherapy. However, this remains contradictory in many cases 


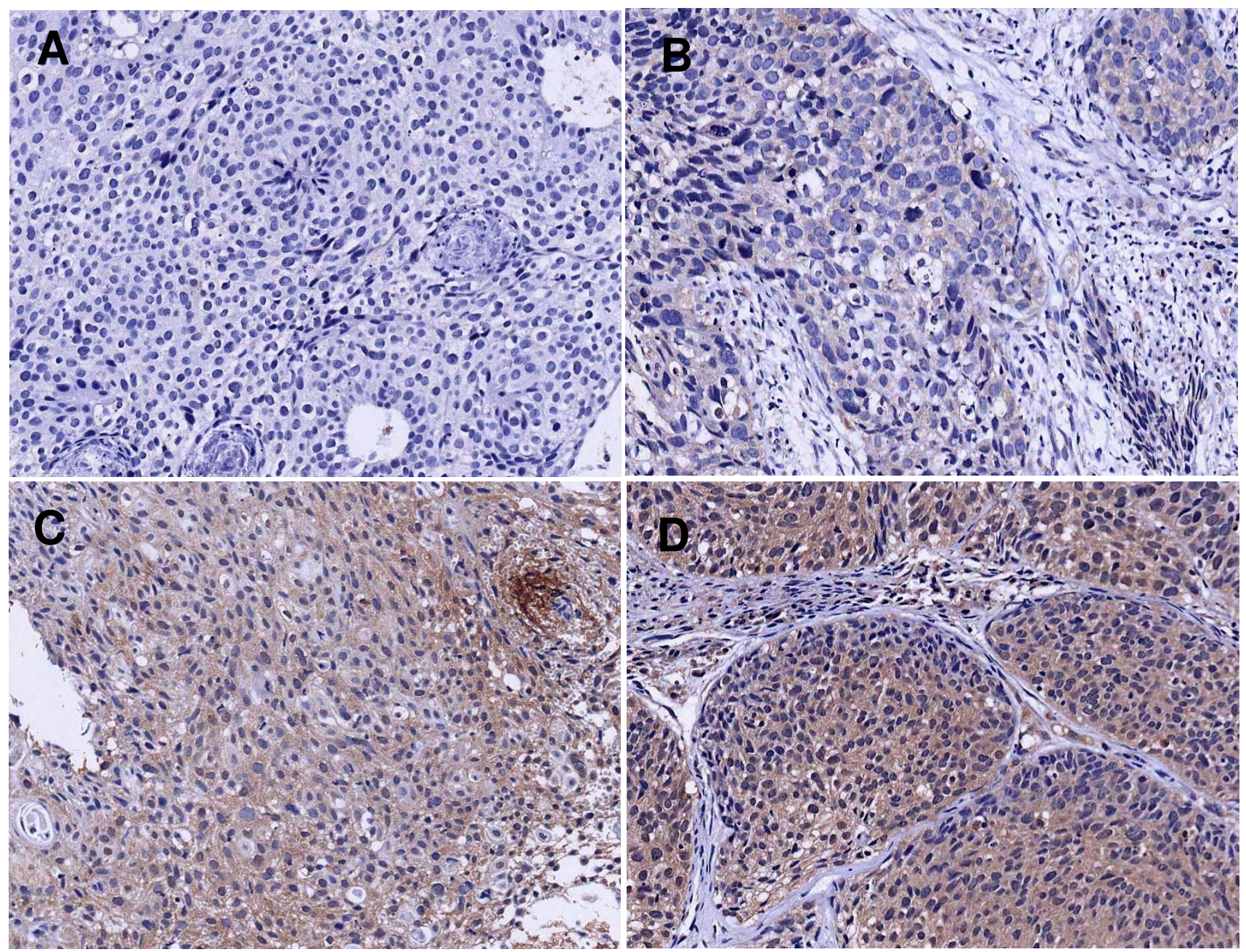

\section{Figure I}

Representative examples of VEGF staining in bulky cervical carcinoma showing cases with no staining (A), weak staining (B), moderate staining (C) and strong staining (D) $(\times 200)$.

$[16,17]$. Unlike normal blood vessels, tumor vessels are structurally and functionally abnormal, i.e., the formation of tortuous and saccular blood vessels that are poorly organized and hyperpermeable [18]. These abnormalities can increase resistance to blood flow and impair blood supply, and therefore compromise the delivery and effectiveness of conventional cytotoxic therapies [19]. It has also been suggested that the role of angiogenic factors in the treatment response may be governed by hypoxia. Piret and colleagues suggested that HIF-1 $\alpha$ (key proteins regulating angiogenesis) has both pro- and anti-apoptotic effects [20]. Mild hypoxia causes the expression of various anti-apoptotic proteins, whereas severe hypoxia leads to cell death, at least in part, through stabilization of p53 by HIF-1 $\alpha$ [20]. The overall balance of the activation effects of HIF- $1 \alpha$ may depend on the type of cancer and treatment modality used [21].
Clinical studies on the correlation between VEGF expression and prognosis have reported inconsistent results [7$9,22]$. Some reasons for the conflicting results include the following. First, the patient groups studied were heterogeneous in terms of disease stages and treatment modalities. Second, the studies were prone to sampling errors because the neovascularization status of the tumor could not be reliably determined with a single measurement of only a small portion of the whole tumor. Jain reported that not only does the microcirculation vary from one tumor to the next, but within the same tumor, it varies both spatially and temporally [23].

\section{Conclusion}

The present study showed that the assessment of VEGF expression in pretreatment biopsy specimens could provide additional information to identify patients with a 
Table 2: Clinicopathologic parameters and the expression of VEGF as predictors of response to neoadjuvant chemotherapy in patients with bulky cervical carcinoma

\begin{tabular}{|c|c|c|c|c|c|c|c|}
\hline \multirow[b]{2}{*}{ Characteristics } & \multirow[b]{2}{*}{ No. of patients } & \multirow[b]{2}{*}{$\mathrm{RR}$} & \multicolumn{2}{|c|}{ Pathologic responder } & \multirow{2}{*}{$\begin{array}{c}\text { Nonresponder } \\
\qquad(n=2 I)\end{array}$} & \multirow[b]{2}{*}{$\mathrm{pb}$} & \multirow[b]{2}{*}{$P c$} \\
\hline & & & $C R(n=4)$ & $\operatorname{OPR}^{a}(n=4)$ & & & \\
\hline Age, years & & & & & & 0.16 & \\
\hline Median & & & 58 & 47 & 46 & & \\
\hline Range & & & $38-60$ & $34-60$ & $33-70$ & & \\
\hline Cervical tumor size $(\mathrm{cm})$ & & & & & & 0.07 & 0.22 \\
\hline Median & & & 3.8 & 3.5 & 5.0 & & \\
\hline Range & & & $3.2-6.0$ & $3.0-5.6$ & $3.7-7.1$ & & \\
\hline Clinical node involvement & & & & & & 0.98 & 0.22 \\
\hline Yes & 11 & $27.3 \%$ & 2 & 1 & 8 & & \\
\hline No & 18 & $27.8 \%$ & 2 & 3 & 13 & & \\
\hline Stage & & & & & & 0.67 & 0.54 \\
\hline IB2 & 20 & $30.0 \%$ & 2 & 4 & 14 & & \\
\hline$\| A$ & 9 & $22.2 \%$ & 2 & 0 & 7 & & \\
\hline SCC Ag level & & & & & & 0.47 & 0.36 \\
\hline$\geq 5 \mathrm{ng} / \mathrm{ml}$ & 14 & $21.4 \%$ & 1 & 2 & 11 & & \\
\hline$<5 \mathrm{ng} / \mathrm{ml}$ & 15 & $33.3 \%$ & 3 & 2 & 10 & & \\
\hline VEGF expression & & & & & & 0.009 & 0.032 \\
\hline Negative & 14 & $50.0 \%$ & 3 & 4 & 7 & & \\
\hline Positive & 15 & $6.7 \%$ & 1 & 0 & 14 & & \\
\hline
\end{tabular}

aResidual tumor but only with less than $3 \mathrm{~mm}$ stromal invasion

bRank sum test or Chi-square test/Fisher's exact test

cLogistic regression analysis with stage, tumor size, SCC-Ag level, and lymph node involvement as a covariate.

$R R$, pathologic response rate; $C R$, complete response; OPR, optimal pathologic response

poor chance of response to NAC and unfavorable prognosis in patients with bulky cervical carcinoma.

\section{Competing interests}

The authors declare that they have no competing interests.

\section{Authors' contributions}

CHC and SYS collected the data, performed analysis and prepared the manuscript. JJC, YAP, HK and TJK performed the immunoassays and statistical analysis. JWL, BGK and JHL selected the cases and interpreted the results. DSB designed the study concept, interpreted the results and approved the final manuscript.

Table 3: Univariate and multivariate analysis of clinicopathologic factors affecting disease-free survival rate

\begin{tabular}{|c|c|c|c|c|c|}
\hline \multirow[b]{2}{*}{ Characteristics } & \multirow[b]{2}{*}{ No. of patients } & \multirow[b]{2}{*}{ 5-y DFS (\%) } & \multirow{2}{*}{$\begin{array}{c}\text { Univariate analysis } \\
P\end{array}$} & \multicolumn{2}{|c|}{ Multivariate analysis } \\
\hline & & & & Relative risk $(95 \% \mathrm{Cl})$ & $P$ \\
\hline \multicolumn{6}{|c|}{ Cervical tumor size } \\
\hline$\geq 5 \mathrm{~cm}$ & 14 & 69.2 & 0.50 & 0.66 & $0.53^{\mathrm{a}}$ \\
\hline$<5 \mathrm{~cm}$ & 15 & 80.0 & & $(0.18-2.42)$ & \\
\hline \multicolumn{6}{|c|}{ Clinical node involvement } \\
\hline Yes & II & 70.6 & 0.49 & 2.30 & 0.39 \\
\hline No & 18 & 81.8 & & $(0.35-15.10)$ & \\
\hline \multicolumn{6}{|l|}{ Stage } \\
\hline IB2 & 20 & 73.3 & 0.83 & 0.67 & 0.64 \\
\hline$\| \mathrm{A}$ & 9 & 77.8 & & $(0.13-3.58)$ & \\
\hline \multicolumn{6}{|l|}{ SCC Ag level } \\
\hline$\geq 5 \mathrm{ng} / \mathrm{ml}$ & 14 & 69.2 & 0.50 & 1.03 & $0.45^{\mathrm{a}}$ \\
\hline$<5 \mathrm{ng} / \mathrm{ml}$ & 15 & 80.0 & & $(0.95-1.12)$ & \\
\hline \multicolumn{6}{|l|}{ VEGF expression } \\
\hline Negative & 14 & 92.3 & 0.033 & 11.40 & 0.037 \\
\hline Positive & 15 & 60.0 & & $(1.15-112.58)$ & \\
\hline
\end{tabular}

a Treated as a continuous variable. 

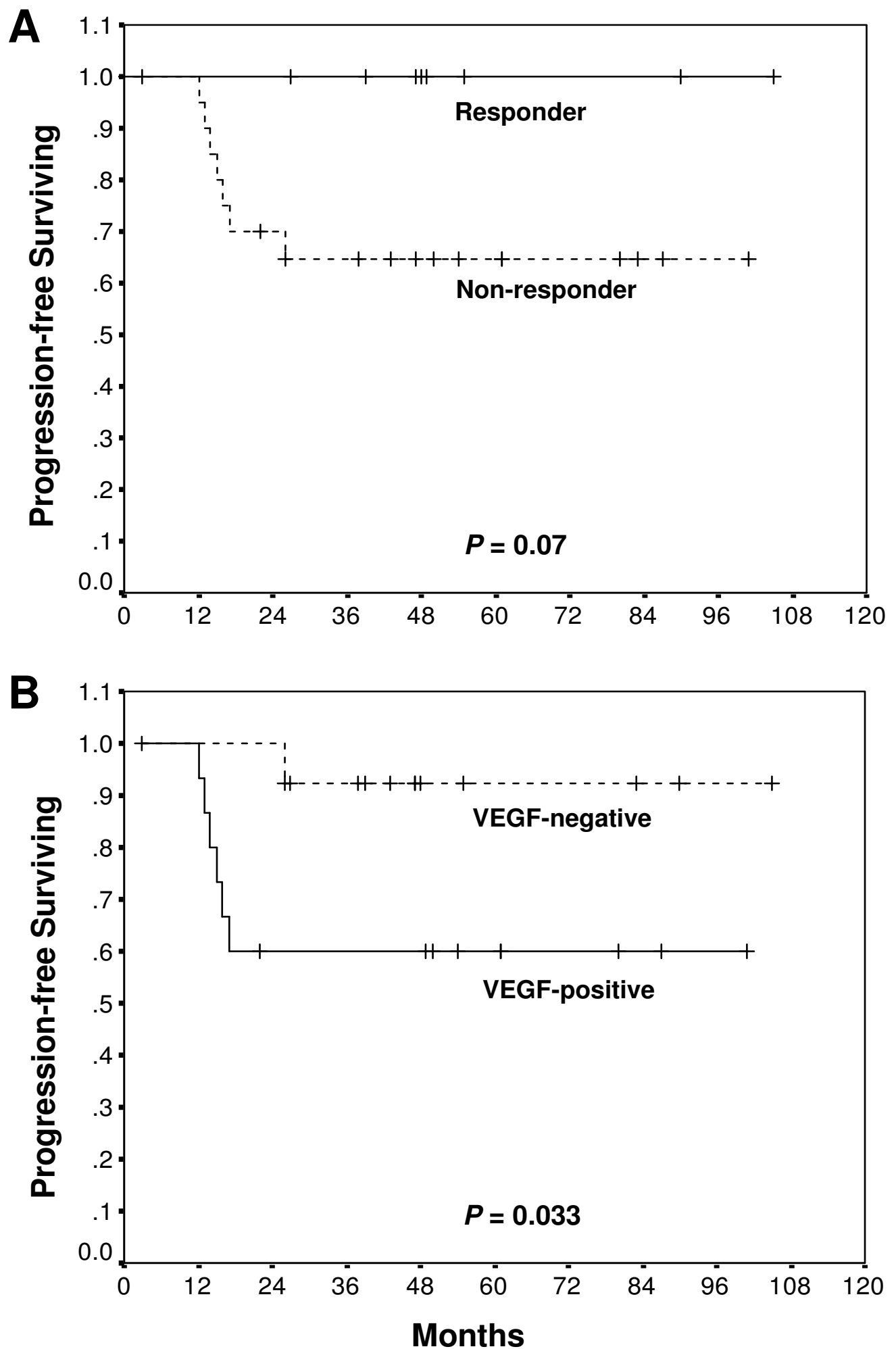

Figure 2

Disease-free survival curve as a function of pathologic response $(A)$ and immunoreactivity to VEGF (B). The $P$ values were determined using the log-rank test. 


\section{Additional material}

\section{Additional file 1}

Negative controls for VEGF staining. All slides show negative staining $(\times 200)$.

Click here for file

[http://www.biomedcentral.com/content/supplementary/14712407-8-295-S1.ppt]

\section{Acknowledgements}

This study was supported by a grant of the Korea Health 2I R\&D Project, Ministry of Health \& Welfare, Republic of Korea (04I2-CR0I-0704-000I)

\section{References}

I. Thomas GM: Improved treatment for cervical cancer - concurrent chemotherapy and radiotherapy. N Engl J Med 1999, 340( I 5): I I98-1200.

2. Pignata S, Silvestro G, Ferrari E, Selvaggi L, Perrone F, Maffeo A, Frezza P, Di Vagno G, Casella G, Ricchi P, et al.: Phase II study of cisplatin and vinorelbine as first-line chemotherapy in patients with carcinoma of the uterine cervix. J Clin Oncol 1999, I 7(3):756-760.

3. Serur E, Mathews RP, Gates J, Levine P, Maiman M, Remy JC: Neoadjuvant chemotherapy in stage IB2 squamous cell carcinoma of the cervix. Gynecol Oncol 1997, 65(2):348-356.

4. Brown LF, Berse B, Jackman RW, Tognazzi K, Guidi AJ, Dvorak HF, Senger DR, Connolly JL, Schnitt SJ: Expression of vascular permeability factor (vascular endothelial growth factor) and its receptors in breast cancer. Hum Pathol 1995, 26(I):86-91.

5. Fine BA, Valente PT, Feinstein GI, Dey T: VEGF, flt-I, and KDR/ flk-I as prognostic indicators in endometrial carcinoma. Gynecol Oncol 2000, 76(I):33-39.

6. Seo Y, Baba H, Fukuda T, Takashima M, Sugimachi K: High expression of vascular endothelial growth factor is associated with liver metastasis and a poor prognosis for patients with ductal pancreatic adenocarcinoma. Cancer 2000, 88( I 0):2239-2245.

7. Cheng WF, Chen CA, Lee CN, Chen TM, Hsieh FJ, Hsieh CY: Vascular endothelial growth factor in cervical carcinoma. Obstet Gynecol 1999, 93(5 Pt I):761-765.

8. Cheng WF, Chen CA, Lee CN, Wei LH, Hsieh FJ, Hsieh CY: Vascular endothelial growth factor and prognosis of cervical carcinoma. Obstet Gynecol 2000, 96(5 Pt I):721-726.

9. Loncaster JA, Cooper RA, Logue JP, Davidson SE, Hunter RD, West $C M$ : Vascular endothelial growth factor (VEGF) expression is a prognostic factor for radiotherapy outcome in advanced carcinoma of the cervix. Br J Cancer 2000, 83(5):620-625.

10. Veikkola T, Karkkainen M, Claesson-Welsh L, Alitalo K: Regulation of angiogenesis via vascular endothelial growth factor receptors. Cancer Res 2000, 60(2):203-2I2.

II. Choi CH, Kim TJ, Lee JW, Kim BG, Lee JH, Bae DS: Phase II study of neoadjuvant chemotherapy with mitomycin-c, vincristine and cisplatin (MVC) in patients with stages IB2-IIB cervical carcinoma. Gynecol Oncol 2007, I 04(I):64-69.

12. Buda A, Fossati R, Colombo N, Fei F, Floriani I, Gueli Alletti D, Katsaros D, Landoni F, Lissoni A, Malzoni C, et al:: Randomized tria of neoadjuvant chemotherapy comparing paclitaxel, ifosfamide, and cisplatin with ifosfamide and cisplatin followed by radical surgery in patients with locally advanced squamous cell cervical carcinoma: the SNAPO I (Studio Neo-Adjuvante Portio) Italian Collaborative Study. J Clin Oncol 2005, 23( I 8):4|37-4|45.

13. Ribeiro U Jr, Finkelstein SD, Safatle-Ribeiro AV, Landreneau RJ, Clarke MR, Bakker A, Swalsky PA, Gooding WE, Posner MC: p53 sequence analysis predicts treatment response and outcome of patients with esophageal carcinoma. Cancer 1998, 83(I):7-18.

14. Kimura S, Kitadai Y, Tanaka S, Kuwai T, Hihara J, Yoshida K, Toge T, Chayama K: Expression of hypoxia-inducible factor (HIF)I alpha is associated with vascular endothelial growth factor expression and tumour angiogenesis in human oesophageal squamous cell carcinoma. Eur J Cancer 2004, 40( I 2): | 904- I 912.

15. Klement G, Baruchel S, Rak J, Man S, Clark K, Hicklin DJ, Bohlen P, Kerbel RS: Continuous low-dose therapy with vinblastine and VEGF receptor-2 antibody induces sustained tumor regression without overt toxicity. J Clin Invest 2000, I05(8):R I5-24.

16. Gasparini G, Bonoldi E, Viale G, Verderio P, Boracchi P, Panizzoni GA Radaelli U, Di Bacco A, Guglielmi RB, Bevilacqua P: Prognostic and predictive value of tumour angiogenesis in ovarian carcinomas. Int J Cancer 1996, 69(3):205-2II.

17. Raspollini MR, Amunni G, Villanucci A, Baroni G, Boddi V, Taddei GL: Prognostic significance of microvessel density and vascular endothelial growth factor expression in advanced ovarian serous carcinoma. Int J Gynecol Cancer 2004, I 4(5):8I 5-823.

18. Baish JW, Jain RK: Fractals and cancer. Cancer Res 2000, 60( I4):3683-3688.

19. Jain RK: The next frontier of molecular medicine: delivery of therapeutics. Nat Med 1998, 4(6):655-657.

20. Piret JP, Mottet D, Raes M, Michiels C: Is HIF-Ialpha a pro- or an anti-apoptotic protein? Biochem Pharmacol 2002, 64(56):889-892.

21. Beasley NJ, Leek R, Alam M, Turley H, Cox GJ, Gatter K, Millard P, Fuggle S, Harris AL: Hypoxia-inducible factors HIF-Ialpha and HIF-2alpha in head and neck cancer: relationship to tumor biology and treatment outcome in surgically resected patients. Cancer Res 2002, 62(9):2493-2497.

22. Tjalma W, Weyler J, Weyn B, Van Marck E, Van Daele A, Van Dam P, Goovaerts G, Buytaert P: The association between vascular endothelial growth factor, microvessel density and clinicopathological features in invasive cervical cancer. Eur J Obstet Gynecol Reprod Biol 2000, 92(2):251-257.

23. Jain RK: The Eugene M. Landis Award Lecture 1996. Delivery of molecular and cellular medicine to solid tumors. Microcirculation 1997, 4(I): I-23.

\section{Pre-publication history}

The pre-publication history for this paper can be accessed here:

http://www.biomedcentral.com/1471-2407/8/295/pre pub
Publish with Biomed Central and every scientist can read your work free of charge

"BioMed Central will be the most significant development for disseminating the results of biomedical research in our lifetime. "

Sir Paul Nurse, Cancer Research UK

Your research papers will be:

- available free of charge to the entire biomedical community

- peer reviewed and published immediately upon acceptance

- cited in PubMed and archived on PubMed Central

- yours - you keep the copyright
BioMedcentral 